\title{
Why we need more research into interprofessional education
}

Marian Traynor, Despina Galanouli, John Gardner and Richard Corry

\author{
Accepted for publication in British Journal of Nursing published by MA \\ Healthcare Limited
}

\section{Key phrases}

1. IPE is necessary for improving patient safety as well as ensuring an efficient, modern health care system worldwide.

2. IPE should start early during undergraduate training and become compulsory for early career graduates.

3. Simulation based IPE should be viewed as a strategy for stakeholders to become mutually responsible for patient safety.

4. There is a need to provide evidence on returns on investment if we are to continue to support Simulation based IPE.

\section{INTRODUCTION}

Teamwork is indisputably a 'good thing' in every walk of professional life, with the exception perhaps of individual endeavours in a singles sports context. Collaboration between healthcare providers is often highlighted as a way of addressing several issues seen in healthcare settings, such as complex care needs arising in the ageing population, increased specialisms in healthcare and unequal distribution of the healthcare services delivered (Higgs et al, 2014). The benefits to be had from the melding of different skills and knowledge, the cross-fertilization of ideas and expertise for solving problems, for innovative developments and for increased efficiency are difficult to challenge. The concept of a multi-professional case meeting borrows heavily on the benefits of harnessing a wide repertoire of skills and expertise from different types of professionals to come up with effective treatment plans for patients with complex problems. However, very often poor communication between professionals of different specialties has been identified as a leading cause for 
adverse incidents in health care in the UK (Department of Health, 2004) and abroad (WHO, 2007; The Joint Commission, 2015). Interprofessional education (IPE) has long been considered an important means of addressing barriers to the harmony of different professionals working together towards the same goal, much as the diverse talents in an orchestra must learn to work together to hone their collective performance into something much more attractive and effective than a mere sum of its parts.

IPE has been defined as 'occasions when two or more professions learn with, from and about each other to improve collaboration and quality of care' (CAIPE, 2002) and has been hailed as necessary for improving patient safety as well as ensuring an efficient, modern health care system worldwide. The WHO and its partners acknowledge that, after almost fifty years of research, the evidence sufficiently indicates that effective IPE enables effective collaborative practice $(\mathrm{WHO}, 2011)$. (Empirical studies nationally and internationally confirm that students who have been exposed to IPE pre-qualification experiences become more confident in their communication and interprofessional relationships (Wilhelmsson et al, 2013) and also more respectful of other professions (Gilligan et al, 2014).

However, it is clear that not just any IPE activity can have the desired outcome. When the term IPE refers merely to lectures attended by more than one discipline, little or no engagement takes place between participants and the experience is primarily a didactic one (Gilligan et al, 2014). Even when IPE experiences do happen in clinical settings, they may be ineffective unless they are structured and well planned. According to Gilligan and his colleagues, the best example of effective IPE in Australia is a six bed student training ward which operates within a 26-bed general medical ward at a hospital, with final year students from various health disciplines undertaking all duties in this training ward as an interprofessional team. According to the authors, however, the biggest challenge facing IPE is the interprofessional culture of the clinical setting, which needs genuine interaction and collaboration between professionals to take place. Ensuring these interprofessional cultural conditions are met requires us to have 
good insights into the educational and value systems of the participants, and the personal factors which make up each professional culture. Hall (2005) argues that such insights can help those designing interprofessional education initiatives to strengthen collaborative practice and shape the interprofessional culture of the clinical setting. Without them, and without the better planning of IPE that they can facilitate, the literature suggests that relationships between professionals may be based on unhelpful stereotypes that can have a negative impact in the clinical setting (see, for example, Rosenstein, 2002; Yong Wang, and Yong-fang Liu et al, $2015 ;$;

Boyce et al (2009) report on another interesting initiative to ensure successful IPE. The University of Queensland's Health Care Team Challenge is an activity that engages all stakeholders including students, academics, practitioners and policymakers in a holistic approach to IPE where 'interprofessional student teams compete at a live public event for a cash prize for the best management plan centred on a complex clinical study' ( $p$ 433). Similar activities have been recorded in Canada, in British Columbia (Boyce et al, 2009), and although they are not a replacement for consistent, curriculum-integrated IPE, they do raise the profile of IPE in health care disciplines, with the potential to contribute to a change of culture in the clinical setting through increased public exposure to interprofessional collaboration.

The research above and a steady stream of systematic reviews in this field going back 15 years (see, for example, Barr et al, 2014; Reeves et al, 2013, Reeves et al, 2010; Hammick et al, 2007; Clifton et al, 2007; Zwarenstein et al, 2005; and Cooper et al, 2001) show that, despite several empirical studies in IPE nationally and internationally there is still a lot of research needed to ensure that successful IPE strategies are designed and implemented.

\section{$\underline{\mathrm{IPE} \text { and the use of High Fidelity Simulation }}$}

Many respondents participating in research on IPE have valued experiences which have taken place in clinical, real-time settings (Gilligan et al, 2014; Bennett et al, 2011) and which are 'authentic and occurred in environments that valued patient quality and safety over hierarchy' 
(Loversidge \& Demb 2015, p303). Hammick et al (2007), in particular, identified this as a key message of an earlier best-evidence systematic review of IPE:

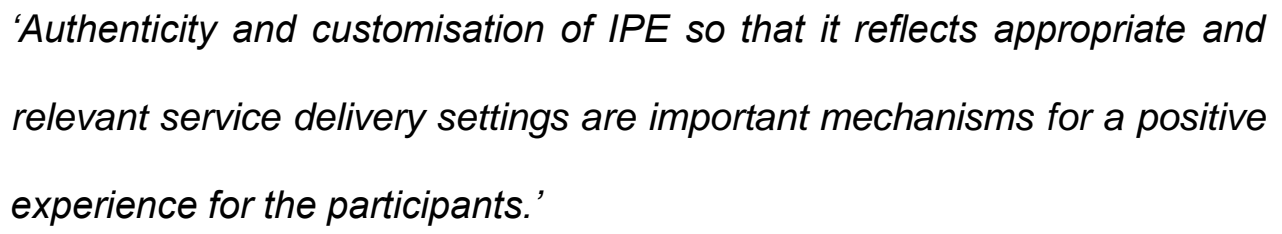

Hammick et al, 2007, p 748

However, with patient safety and comfort in mind, real-time clinical settings may not always be a realistic aspiration for IPE especially in acute care, in the operating theatre or even in palliative care. In such cases using simulation can be the best alternative available and research from the UK (Watters et al. 2015), Canada (Jones-Bonofiglio and Kortes-Miller, 2012; Baker et al, 2008), the US (Shoemaker et al, 2015; Paige et al, 2014; Smithburger et al, 2013; Booth \& McMullen-Fix, 2012) and Singapore (Liaw et al, 2014) shows that it can be as challenging, rewarding and effective as a real-life scenario.

\section{The UK context}

IPE in the UK began in the 1960s with the first recorded IPE initiative taking place in 1966 (Barr, 2007). Fifty years later, interest in IPE has been steadily growing and has been given added impetus by several high profile malpractice cases, for example as reported in the Bristol Royal Infirmary inquiry which took place in 2001. The subsequent government response to this inquiry included a recommendation that ' ...there should be more opportunities for different health care professions to share learning and that more emphasis should be placed upon the non-clinical aspects of care, such as communication skills, in the education, training and development of those working within the NHS' (DOH 2002, p10).

The Centre for the Advancement of Interprofessional Education (CAIPE) is an important agent in the promotion of IPE in the UK and was founded in 1987 to develop shared learning among 
professions, encompassing all fields of public health and social care. Since its foundation, it has been at the forefront of promoting IPE, providing information and advice through its website and various publications, seminars and workshops. For example, in their latest review of pre-qualifying interprofessional education in the UK, Barr and his colleagues offered a set of recommendations for various bodies ranging from regulatory to teaching to 'promote growth and effectiveness, especially capacity in learning and teaching, and to strengthen the infrastructure to remove the problems of alignment and enhance work-based IPE' (Barr et al, 2014, p 5). In addition to the work of CAIPE in promoting IPE, the World Health Organisation (WHO) is currently leading a global drive to build on patient safety education and has published a comprehensive guide to implementing multi professional patient safety education (WHO, 2011).

The commitment to IPE shown by teaching and regulatory bodies is clear but the available research demonstrates a tendency to focus initiatives in the pre-registration phase (see, for example, Liaw et al, 2014; Paige et al, 2014; Smithburger et al, 2013; Booth \& McMullen-Fix, 2012; Stewart et al, 2010; Baker et al, 2008) with only a few studies examining IPE involving post-qualification doctors and nurses (for example Watters et al, 2015; Paige et al, 2009). The challenges involved in conducting such research with working professionals may include busy ward timetables, funding constraints and ethical approval.

There is some evidence that IPE interventions at undergraduate level do not always survive the transition to the work environment and do not have the expected outcomes regarding communication and collaboration at the clinical setting (Wilhelmsson et al, 2013). Investing mostly in pre-registration IPE could arguably, therefore, be ineffective if there is no followthrough to consolidate the benefits gained into the early years of clinical practice and beyond. More studies are needed to explore how IPE in general and simulation-based IPE in particular at post-graduate level can contribute to better quality interaction between healthcare professionals and better patient outcomes. One small scale IPE simulation study is reported below as a taster for the sorts of outcomes that could be gained from a more comprehensive study. 


\section{The Potential for IPE Simulation Studies}

In 2011, one UK higher education institution conducted a small-scale, descriptive study on the usefulness of high fidelity ${ }^{1}$ (HF) simulation in a postgraduate interprofessional education programme for recently qualified doctors and nurses in an acute care setting. The aim of the study was to show if an HF simulator could enhance a post-graduate interprofessional education session on team-working and communication skills. Ethical approval was sought from the NI Office for Research Ethics and was granted prior to advertising the study in hospital staff rooms' noticeboards. Participants were selected on the basis of being (a) qualified for at least 12 months and (b) working in an acute clinical setting where real-life IPE activities could be more difficult to plan due to patient safety concerns (necessitating an HF simulation as an alternative).

\section{$\underline{\text { METHODS }}$}

\section{$\underline{\text { Sample }}$}

Six doctors $(4 \mathrm{xf}, 2 \mathrm{xm})$ and six nurses $(5 \mathrm{xf}, 1 \mathrm{xm})$ volunteered for the study and their consent was sought through a conventional participant information sheet (PIS) and informed consent form. The PIS made reference to the fact that in the event of any evidence of unsafe practice during the scenarios, their manager would be informed. All six doctors were aged between 22 and 27 and the nurses were variously aged between 22 and 27 (2), 28 and 32 (3) and 33 and 39 (1). Seven volunteers participated in two focus group discussions; one group comprised a nurse and a doctor and the other had two doctors and three nurses.

\footnotetext{
${ }^{1}$ The term high fidelity in simulation describes the level of technology of the patient- manikin. A highfidelity simulator manikin as used in this session, is designed and equipped with technology to replicate many physiological functions (e.g. interaction, breath sounds, blinking etc.) and human anatomical features. Low-fidelity manikins, by contrast, cannot interact and exhibit little or no technology, e.g. Resusci-Annie used for BLS (Basic Life Support).
} 


\section{Methodology}

On the day of the study the 12 volunteers completed a pre-session questionnaire to ascertain their previous experience and knowledge of high-fidelity simulation and interprofessional education. They were then asked to participate in teams of one nurse and one doctor in a typical clinical case scenario using an HF simulator. The scenario took place in a simulation suite comprising a patient simulator and one-way mirror. All of the sessions were videorecorded for use in the debriefing sessions. The participants also completed a post-session questionnaire.

\section{$\underline{\text { Instruments }}$}

The pre- and post-questionnaires comprised two parts; the first part included questions on the age, gender and profession of the participants, any previous experience with IPE/shared learning activities and their motivation to participate in this study. The second part consisted of a Likert scale featuring 35 items for the pre-questionnaire and 44 items for the postquestionnaire. A number of statements were the same for both questionnaires in order to examine any change in attitudes brought about by the HF simulation session.

\section{$\underline{\text { RESULTS }}$}

The descriptive results from the questionnaire responses indicated that the IPE session had at least a modest positive effect on the participating doctors with all six agreeing the benefits of collaboratively developing team working skills after the sessions compared to four beforehand. The six nurses had perceived the benefits before they undertook the sessions and they retained this view.

Table 1 presents these statements from the pre-questionnaire that attracted consensus from all participants: 
The post-questionnaire comprised items on the use of HF simulation in the IPE session they had just undertaken and on their views on further IPE training using HF simulators. All respondents agreed or strongly agreed with the statements in Table 2 and despite the sample being very small there is an encouraging confirmation of the potential of this type of interprofessional education.

The open comments provided by the participants in the post-session questionnaire and the focus groups were also favourably disposed to the IPE simulation process. Empathising with other professions and overcoming problems arising from perceived hierarchies were among the advantages mentioned. The participants felt that the IPE simulation activity impacted positively on the interactions between professionals and held out the potential for changing long-held practices. A selection of the comments illustrates these views and signals the potential that IPE simulations have:

Empathise with other professions, learn more about their roles and responsibilities; all dealing with the same patients, why not learn together? Overcome hierarchy in hospital, it's a massive problem. (Doctor)

Left course feeling as if practice can be changed. (Nurse)

One doctor felt that recording the process could be off-putting for some individuals while two others pointed to differences that may exist in clinical equipment and knowledge-bases for people from different hospitals; and how this could affect collaboration during the IPE simulation session. Communication was key:

I think [the course] is also a breakdown of barriers... Everyone is there for the main cause that is the patient and this makes it easier for people to communicate with each other. (Doctor)

Realising that everyone has the same fears; that everyone is afraid of appearing stupid or stepping on someone's toes and realising that actually everyone is 
afraid of that... and know that everyone is on the same boat and it helps everybody, it's great... (Doctor)

Relatively speaking everyone who was here today was pretty good at communicating whereas there are some people I have worked with that would definitely benefit from a bit of communication skills teaching. (Nurse)

It is also clear from the participants' comments that they believe that practice does improve through IPE with increased respect and understanding of each other's roles, more effective engagement with each other, and bringing about better care and improved outcomes for patients:

It gives nurses and doctors a chance to work together... you definitely learn a lot about how doctors think and I am amazed to see, or to know rather, literally about how little actually doctors are aware of nursing limitations and even our perception of how we are viewed. I am feeling now from doing this course and from speaking to other doctors that they actually hold us in higher esteem than we actually think they do... (Nurse)

I think [IPE simulation] is really good for situations like medical emergencies, it's really useful to know what role everyone has... As a medical student you do everything and it is useful to know what nurses do and get used to communicating and handing over and getting feedback ... (Doctor)

All of the participants agreed that all aspects of the IPE activity, the high fidelity simulation, the scenarios and the debriefing were 'great' and 'effective' in attracting and maintaining interest and attention. Specifically the reflective debriefing, the use and playback of the video footage and also the positive, constructive feedback seemed to be important factors in the success of the activity. All the participants agreed that further such training should be introduced to promote interprofessional collaboration and that it should start early during undergraduate training, becoming compulsory for early career graduates as the realities of the transition from 
an academic to a professional clinical setting may not allow for new graduates to get involved voluntarily in such initiatives.

\section{DISCUSSION AND CONCLUDING REMARKS}

This study serves as a pilot outlining the key areas that need to be pursued through research. These include the need to explore how nurses' and doctors' perceptions of each other's roles can be highlighted in IPE simulations, how their value systems affect them in clinical setting and in collaborative engagement with each other. More research is needed to identify how communication and interaction between nurses and doctors can be optimized through training in simulation based education, primarily for better patient outcomes but also to foster an efficient and harmonious working culture. The study has yielded valuable information on the design of the instruments and the processes of group feedback and individual reflective debriefings using video recordings of practice. Self-reporting is at the centre of this small study but future research needs to be more specifically linked to patient-related impact and outcomes to ensure that patient safety is at the heart of the IPE training objectives and there have been recent calls for such strengthening of the evidence base of IPE research (see Cox et al, 2016). Also, that the concepts of teamwork and communication between professionals within any IPE implementation are consistent with new GMC standards for medical education and training (GMC, 2015) and the Patient Safety Curriculum Guide published by the World Health Organisation (WHO, 2011). Looking to the future, it is clear that a step change in the design, frequency and spread of use of high fidelity IPE simulations requires much more sophisticated research evidence, particularly given the resources needed to deliver such a programme of study. Such a research study would provide the much needed evidence on the impact of IPE on patient care and it would ultimately begin to unlock the interactional complexities that underpin the successful working together of various professionals, in that same fluent manner as multi-instrument orchestras, for the benefit of patients. 


\section{Acknowledgements}

Our special thanks go to the doctors and nurses for their participation and insights.

\section{REFERENCES}

Baker, C., Pulling, C., McGraw, R., Dagnone, J.D., Hopkins-Rosseel, D. \& Medves, J. 2008. Simulation in interprofessional education for patient-centred collaborative care. $J A d v$ Nurs, 64, 372-379.

Barr, H., Helme, M. \& D'Avray, L. 2014. Review of Interprofessional Education in the United Kingdom 1997-2013. Centre for Advanced of Interprofessional Education (CAIPE). Fareham. Accessed on 23 February 2016 at http://caipe.org.uk/silo/files/iperg-review15-4-14-with-links-pdf.pdf.

Barr, H. 2007. Interprofessional education in the United Kingdom. Some historical perspectives 1966-1996. A supplement to: creating an interprofessional workforce: an education and training framework for health and social care. Centre for the Advancement of Interprofessional Education, London.

Bennett, P.N., Gum, L., Lindeman, I., Lawn, S., McAlister, S., Richards, J., Kelton, M. \& Ward, H. 2011. Faculty perceptions of interprofessional education. Nurse Educ Today, 31, 571-576.

Booth, T.L. \& McMullen-Fix, K. 2012. Collaborative Interprofessional Simulation in a Baccalaureate Nursing Education Program. Nurs Educ Perspect, 33,127-129.

Boyce, R.A., Moran, M.C., Nissen, L.M., Chenery, H.J. \& Brooks, P.M. 2009. Interprofessional education in health sciences: the University of Queensland Health Care Team Challenge. Med J Aust, 190, 433-436.

CAIPE. Centre for the Advancement of Interprofessional Practice and Education. 2002. Accessed on 23 February 2016 at http://caipe.org.uk/resources/defining-ipe/.

Cooper, H., Carlisle, C., Gibbs, T. \& Watkins, C. 2001. Developing an evidence base for interdisciplinary learning: a systematic review. J Adv Nurs, 35, 228-237.

Cox, M., Cuff, P., Brandt, B., Reeves, S. \& Zierler, B. 2016. Measuring the impact of interprofessional education on collaborative practice and patient outcomes, J Interprof Care, 30, 1-3, DOI: 10.3109/13561820.2015.1111052.

Department of Health 2002. Learning from Bristol: the Department of Health's Response to the Report of the Public Inquiry into children's heart surgery at the Bristol Royal 
Infirmary 1984-1995. Department of Health. HMSO:Norwich. Accessed on 1 February 2016 at:

https://www.gov.uk/government/uploads/system/uploads/attachment data/file/273320/5 363.pdf

Department of Health (2004) The NHS improvement plan: Putting people at the heart of public services, London: Department of Health.

Faresjö, T., Wilhelmsson, M., Pelling, S., Dahlgren, L.O. \& Hammar, M. 2007. Does interprofessional education jeopardize medical skills? J Interprof Care, 21, 1-4.

Gilligan, C., Outram, S. \& Levett-Jones, T. 2014. Recommendations from recent graduates in medicine, nursing and pharmacy on improving interprofessional education in university programs: a qualitative study. BMC Med Educ, 14, 52.

GMC. General Medical Council. 2015. Promoting excellence: standards for medical education and training. Accessed on 23 February 2016 at: http://www.gmcuk.org/Promoting excellence standards for medical education and training 0715.pdf 61939165.pdf

Hall, P. 2005. Interprofessional teamwork: Professional cultures as barriers. J Interprof Care, 19, sup1, $188-196$.

Hammick, M., Freeth, D., Koppel, I., Reeves, S. \& Barr, H. 2007. A best evidence systematic review of interprofessional education: BEME Guide no. 9. Med Teach, 29, 735-751.

Higgs, J., Croker, A., Tasker, D., Hummell, J. and Patton, N. (2014) Health Practice Relationships, Rotterdam: Sense Publishers.

Jones-Bonofiglio, K. \& Kortes-Miller, K. 2012. Dying with Grace: A pilot project exploring the use of high-fidelity simulation in interprofessional palliative care education. Canadian Virtual Hospice. Accessed on 27/1/2016 on: http://tinyurl.com/znls2k5.

Liaw, S.Y., Zhou, W.T., Lau, T.C., Siau, C. \& Chan, S.W. 2014. An interprofessional communication training using simulation to enhance safe care for a deteriorating patient. Nurse Educ Today, 34, 259-264.

Loversidge, J. \& Demb, A. 2015. Faculty perceptions of key factors in interprofessional education. J Interprof Care, 29, 298-304.

Paige, J.T., Garbee, D.D., Kozmenko, V., Yu, Q., Kozmenko, L., Yang, T., Bonanno, L. \& Swartz, W. 2014. Getting a Head Start: High Fidelity, Simulation-Based Operating Room Team Training of Interprofessional Students. J Am Coll Surg, 218,140-149.

Paige, J.T., Kozmenko, V., Yang, T., Gururaja, R.P., Hilton, C.W., Cohn, I. \& Chauvin, S.W. 2009. High-fidelity, simulation-based, interdisciplinary operating room team training at the point of care. Surgery, 145,138-146.

Reeves, S., Goldman, J., Burton, A. \& Sawatzky-Girling, B. 2010. Synthesis of Systematic Review Evidence of Interprofessional Education. J Allied Health, 39,198-203. 
Reeves, S., Perrier, L., Goldman, J., Freeth, D. \& Zwarenstein, M. 2013. Interprofessional education: effects on professional practice and healthcare outcomes (update) (Review). The Cochrane Collaboration. Published by John Wiley \& Sons, Ltd.

Rosenstein, AH. 2002. Nurse-Physician Relationships: Impact on Nurse Satisfaction and Retention. Am J Nurs, 102, 26-34.

Shoemaker, M.J., de Voest, M., Booth, A., Meny, L. \& Victor, J. 2015. A virtual patient educational activity to improve interprofessional competencies: A randomized trial. $J$ Interprof Care, 29, 395-397.

Smithburger, P.L., Kane-Gill, S.L., Kloet, M.A., Lohr, B. \& Seybert, A.L. 2013. Advancing interprofessional education through the use of high fidelity human patient simulators. Pharmacy Practice (Granada), 11, 61-65.

Stewart, M., Kennedy, N. \& Cuene-Grandidier, H. 2010. Undergraduate interprofessional education using high-fidelity paediatric simulation. Clin Teach, 7, 90-96.

The Joint Commission 2015. National Patient Safety Goals Effective January 1, 2015. Hospital Accreditation Programme. Oak Brook Illinois. Accessed on 23 February 2016 at: http://www.jointcommission.org/assets/1/6/2015 npsg hap.pdf

Watters, C., Reedy, G., Ross, A., Morgan, N., Handslip, R. \& Jaye, P. 2015. Does interprofessional simulation increase self-efficacy: a comparative study. BMJ Open, 5, e005472. doi:10.1136/ bmjopen-2014-005472.

WHO. World Health Organisation. 1978. Declaration of Alma-Ata. International Conference on Primary Health Care. Alma-Ata, USSR.

WHO. World Health Organisation. 2007. Communication During Patient Hand-Overs. World Health Organization. Geneva. Accessed on 1 February 2016 at: http://www.who.int/patientsafety/solutions/patientsafety/PS-Solution3.pdf

WHO. World Health Organisation. 2011. Patient Safety Curriculum Guide. Multi-professional Edition. Geneva. Accessed on 23 February 2016 at: http://apps.who.int/iris/bitstream/10665/44641/1/9789241501958 eng.pdf.

World Health Organisation (2011) Framework for Action on Interprofessional Education and Collaborative Practice, Geneva: World Health Organisation.

Wilhelmsson, M., Svensson, A., Timpka, T. \& Faresjö, T. 2013. Nurses views of interprofessional education and collaboration: A comparative study of recent graduates from three universities. $J$ Interprof Care, 27, 155-160.

Yong Wang, Yong-fang Liu, Hao Li \& Tingyu Li. 2015. Attitudes toward Physician-Nurse Collaboration in Pediatric Workers and Undergraduate Medical/Nursing Students. Behav Neurol. doi:10.1155/2015/846498, 
Zwarenstein, M., Reeves, S. \& Perrier, L. 2005. Effectiveness of pre-licensure interprofessional education and post-licensure collaborative interventions. J Interprof Care, 19, sup1, 148-165. 
Patients benefit when doctors and nurses work together

For shared learning to work professions need to trust and respect each other

In healthcare good communication between the professions is essential

Errors in patient management are more likely when doctors and nurses don't work well together

Good communication is an essential skill for every healthcare professional

I would welcome the opportunity for more coaching on communication skills in my PG training

Effective team working between doctors and nurses is not necessary for safe patient care

The ability to work in a team is essential for every healthcare professional

Team working in healthcare could be better

Table 1 Pre-questionnaire statements attracting consensus between doctors and nurses

Overall the high fidelity simulator was a good learning environment for this post-graduate IPE course

Using the simulator kept my interest where other types of teaching might have failed

The simulator environment was good at getting the two professions to work together

The simulator scenarios were an excellent opportunity to practise and critique some of the tips discussed by the introductory session

The simulator scenarios highlighted the importance of good communication and team working

The use and playback of the video footage enhanced the 'debrief' sessions in terms of their educational value

High fidelity simulation and debrief is a good way to practise and learn clinical skills for postgraduates

High fidelity simulation and debrief is an effective way to practise and learn communication skills

High fidelity simulation and debrief is an effective way to practise and learn team working skills

I think that this IPE simulation course will have helped me to work better within the multidisciplinary team in my workplace

There should be more high fidelity simulation in postgraduate training

Table 2 Post-questionnaire statements attracting consensus between doctors and nurses 